\title{
Small Random Perturbations of Dynamical Systems and the Definition of Attractors
}

\author{
David Ruelle \\ Institut des Hautes Etudes Scientifiques, 35, route de Chartres, F-91440 Bures-sur-Yvette, France
}

\begin{abstract}
The "strange attractors" plotted by computers and seen in physical experiments do not necessarily have an open basin of attraction. In view of this we study a new definition of attractors based on ideas of Conley. We argue that the attractors observed in the presence of small random perturbations correspond to this new definition.
\end{abstract}

\section{Introduction}

Let $\left(f^{t}\right)$ be a dynamical system, i.e., a group or semigroup of maps $M \mapsto M$ parametrized by a discrete or continuous time $t$. We assume that $M$ has a topological or differentiable structure, and that the $f^{t}$ are continuous or differentiable. There often exist subsets $\Lambda$ of $M$ which attract neighboring points $x$, this means that $f^{t} x$ tends to $\Lambda$ when $t \rightarrow \infty$. Such subsets $\Lambda$ are called attracting sets or attractors. In the simplest cases $\Lambda$ is an attracting fixed point or periodic orbit. More complicated situations have however been studied, notably Smale's Axiom $A$ attractors (see Smale [35], Bowen [4], and Williams [37]).

Attractors are of interest for the description of the asymptotic behavior of physical systems (or the long term behavior of all kinds of natural phenomena). In particular, some attractors (now called strange attractors) show the phenomenon of sensitive dependence on initial condition, i.e., a small change in initial condition grows exponentially with time (the perturbation need grow only as long as it is small). Landau [20] and Hopf [16] used quasiperiodic attractors ${ }^{1}$ to try to describe hydrodynamic turbulence (these attractors do not exhibit sensitive dependence on initial condition). Lorenz [23] found an attractor with sensitive dependence in approximate convection equations, and suggested that this may explain the difficulty of long term weather predictions. Ruelle and Takens [33] proposed that hydrodynamic turbulence is described by non-quasiperiodic attrac-

1 A quasiperiodic attractor is an attracting torus $T^{m}$ such that the time evolution restricted to $T^{m}$ becomes, in suitable coordinates, a translation with dense orbits 
tors (and introduced the name "strange attractor"). Hydrodynamical experiments by Ahlers [1], Gollub and Swinney [14], and others, favor the explanation of turbulence in terms of strange attractors with sensitive dependence on initial condition; a reasonable understanding of the onset of turbulence has now been obtained in those terms (see Ruelle [29] for a review).

Dynamical systems can be studied by computer. Various new attractors have been found in this way (see Hénon [15], Feigenbaum [10-12]), and they have been much investigated.

In view of the above considerations, it seems desirable to give a precise mathematical definition of attractors, and this is the purpose of the present paper. One would like the definition to satisfy various requirements, and the requirements turn out to be somewhat conflicting. We shall, in Sect. 2, discuss possible conditions which may be imposed in the definition of attracting sets. In Sects. 3 and 4 we shall define attracting sets and attractors, respectively, and we shall study their properties. In Sect. 5 we argue that, under suitable conditions, a dynamical system with small random perturbations asymptotically "lives" on attractors. Section 6 contains some further remarks.

\section{Conditions on Attracting Sets and Attractors}

It is convenient to assume that a metric dist is given on $M$, although in Sect. 3 we shall need only a topology, and in Sect. 4 a uniform structure ${ }^{2}$. The family $\left(f^{t}\right)$ of continuous maps $f^{t}: M \mapsto M$ is indexed ${ }^{3}$ by $\mathbb{Z}, \mathbb{Z}_{+}$(discrete time case), $\mathbb{R}$, or $\mathbb{R}_{+}$ (continuous time case). The group or semigroup properties

$$
f^{0}=\text { identity }, \quad f^{s} \circ f^{t}=f^{s+t}
$$

hold wherever defined. We assume that $(x, t) \mapsto f^{t} x$ is continuous where defined (this is a new requirement only in the continuous time case, and will be used only in Sect. 4). In Theorem 4.4 we shall also require some uniform continuity which is automatically satisfied in applications to differentiable dynamical systems. We now make a list of properties which an attracting set or an attractor $\Lambda$ may want to possess.

(A) Invariance, i.e., $f^{t} \Lambda=\Lambda$ for all $t$.

(B) Attractivity. We may assume that there is a neighborhood $U$ of $\Lambda$ such that for every neighborhood $V$ of $\Lambda, f^{t} U \subset V$ for all sufficiently large $t$. A weaker condition would be that $\Lambda$ has a neighborhood $W$ such that $\lim _{t \rightarrow \infty} \operatorname{dist}\left(f^{t} \dot{x}, \Lambda\right)=0$ for all $x \in W$. We shall use the stronger condition in the definition of attracting sets. Attractors will satisfy an attractivity condition different from the above two.

\section{(C) Closedness or Compactness}

(D) Irreducibility. If an attracting set consists of a number of disjoint invariant pieces, one would like to consider each piece as an attractor, removing "irrelevant"

2 We shall thus in fact use the topology and the uniform structure defined by the metric. For definitions, see for instance Bourbaki [2]

$3 \quad \mathbb{Z}_{+}$and $\mathbb{R}_{+}$are the sets of elements $\geqq 0$ in $\mathbb{Z}$ and $\mathbb{R}$, respectively 
points like (perhaps) wandering points ${ }^{4}$. Examples of irreducibility conditions are: positive transitivity [there is $x \in A$ such that the set of limit points of $\left(f^{t} x\right)_{t>0}$ is $\Lambda$ ] or existence of an ergodic measure with support $\Lambda$. We shall impose a still different condition (chain transitivity) in our definition of attractors.

\section{(E) Unique Decomposability into Irreducible Pieces}

(F) Stability Under Small Perturbations. Experiments are always subject to perturbations, and computer calculations to roundoff errors. Small perturbations will play an essential role in our definition of attractors.

In Sect. 3 we shall define an attracting set by (A)-(C). Attractors will be defined in Sect. 4 so as to permit a natural decomposition of an attracting set into irreducible pieces. For instance the vector field $x \mapsto X(x)=-x^{4} \sin \frac{\pi}{x}$ on $\mathbb{R}$ has the interval $[-1,1]$ as an attracting set, and the corresponding irreducible pieces (attractors) will be the points $\pm \frac{1}{n}$ for $n$ odd and 0 ; note that $\{0\}$ is not an attracting set. Attractors will in fact be limit sets, as time tends to $+\infty$, of perturbed trajectories, and our definition will have the effect that a dynamical system preserving a smooth measure on a connected compact manifold will have the whole manifold as an attractor. In fact for the trivial system $\left(f^{t}\right)$ with $f^{t}=$ identity for all $t$ on a connected manifold, one has the choice of declaring every point to be an attractor, or the whole manifold to be an attractor. We make the latter choice. The necessity of giving a definition of attractors weaker than that of attracting sets is clear from the example of the Feigenbaum Cantor set (see Sect. 6.1); this is an attractor but not an attracting set because it is a limit of (nonattracting) periodic points.

\section{Attracting Sets}

Let $M$ be a Hausdorff topological space and $\left(f^{t}\right)$ a dynamical system (indexed by $\mathbb{Z}, \mathbb{Z}_{+}, \mathbb{R}$, or $\mathbb{R}_{+}$, the $f^{t}$ are continuous $\left.M \mapsto M\right)$. We say that the closed set $\Lambda \subset M$ is an attracting set if it has a neighborhood $U$ such that the condition (a) and one of the conditions $\left.(b),\left(b^{\prime}\right),\left(b^{\prime \prime}\right), b^{\prime \prime \prime}\right)$ below are satisfied.

(a) For every neighborhood $V$ of $\Lambda$, we have $f^{t} U \subset V$ when $t$ is large enough.

(b) $f^{t} \Lambda \supset \Lambda$ when $t$ is large enough.

(b') $f^{t} \Lambda=\Lambda$ for all $t$.

(b") $\bigcap_{t \geqq T} f^{t} U=\Delta$ for some $T$.

$\left(\mathrm{b}^{\prime \prime \prime}\right) \bigcap_{t \geqq T} f^{t} U=\Lambda$ for all $T$.

3.1. Proposition. Given (a), the conditions $(\mathrm{b}),\left(\mathrm{b}^{\prime}\right),\left(\mathrm{b}^{\prime \prime}\right),\left(\mathrm{b}^{\prime \prime \prime}\right)$ are all equivalent. If $U \neq \emptyset$, then $\Lambda \neq \emptyset$.

4 A point $x$ is wandering if it has a neighborhood $N$ such that $f^{t} N \cap N=\emptyset$ for all sufficiently large $t$. A point which is not wandering is called nonwandering: for each $N$ we have $f^{t} N \cap N \neq \emptyset$ for some arbitrarily large $t$ 
(b) $\Rightarrow\left(\mathrm{b}^{\prime}\right)$ and $\left(\mathrm{b}^{\prime \prime \prime}\right)$. For every $T$, (a) yields $\Lambda \supset \bigcap_{t \geqq T} f^{t} U$, while (b) gives 1) $\bigcap_{t \geqq T} f^{t} U$ for large $T$. Thus, for sufficiently large $T, T+\tau$,

$$
\Lambda=\bigcap_{t \geqq T+\tau} f^{t} U \supset f^{\tau} \bigcap_{t \geqq T} f^{t} U=f^{\tau} \Lambda
$$

In particular $f^{\tau} \Lambda \subset \Lambda$ for all $\tau$. If we did not have equality in this inclusion, (b) would be contradicted, therefore we have proved that $\left(b^{\prime}\right)$ holds. Because of this we may now derive (1) for all values of $T$, taking $\tau=0$ proves $\left(\mathrm{b}^{\prime \prime \prime}\right)$.

$\left(\mathrm{b}^{\prime \prime}\right) \Rightarrow(\mathrm{b})$. Given $\tau$, suppose that $x \notin f^{\tau} \Lambda$, then $\Lambda \subset\left(f^{\tau}\right)^{-1}(M \backslash\{x\})$ and, by (a), $f^{t} U \subset\left(f^{\tau}\right)^{-1}(M \backslash\{x\})$ for sufficiently large $t$. Therefore $f^{t} U \subset M \backslash\{x\}$ for sufficiently large $t$, and $\left(\mathrm{b}^{\prime \prime}\right)$ implies that $x \notin \Lambda$. We have proved $f^{\tau} \Lambda \supset \Lambda$, and therefore (b).

$\left(b^{\prime}\right) \Rightarrow(b)$ and $\left(b^{\prime \prime \prime}\right) \Rightarrow\left(b^{\prime \prime}\right)$ obviously. This completes the equivalence proof. If $\Lambda$ were empty, one could take $V=\emptyset$, and this would contradict (a) when $U \neq \emptyset$.

A set $U$ such that the properties (a), (b) are satisfied will be called a fundamental neighborhood of the attracting set $\Lambda$. The open set $W=\bigcup_{t}\left(f^{t}\right)^{-1} U$ is called the basin of attraction of $\Lambda$; $W$ consists of those $x \in M$ such that $f^{t} x \rightarrow \Lambda$ when $t \rightarrow \infty$, and therefore $W$ is independent of the choice of $U$. Notice that if $\left(f^{t}\right)$ is a group we may take $\Lambda$ equal to the whole manifold $M$, and then $\Lambda=U=W$.

3.2. Proposition. If $U$ is open in $M$ and $f^{t} U$ is contained in $U$ and relatively compact for all sufficiently large t, then

$$
\Lambda=\bigcap_{t \geqq 0} f^{t} U
$$

is a compact attracting set with fundamental neighborhood $U$.

By our assumption, if $\tau$ is sufficiently large, the closure $K$ of $f^{\tau} U$ is compact and contained in $U$. Thus

$$
\bigcap_{t \geqq 0} f^{t} K \subset \Lambda \subset \bigcap_{t \geqq \tau} f^{t} U \subset \bigcap_{t \geqq 0} f^{t} K
$$

and therefore

$$
\Lambda=\bigcap_{t \geqq 0} f^{t} K
$$

is compact.

Let $V$ be open, $V \supset \Lambda$. Since $\bigcap_{t \geqq 0} f^{t} K \backslash V=\emptyset$, there will, by compactness, be $t_{1}, t_{2}, \ldots, t_{n}$ such that

$$
f^{t_{1}} K \cap \ldots \cap f^{t_{n}} K \subset V
$$

Thus $f^{t} K \subset V$ for sufficiently large $t$, hence also $f^{t} U \subset V$ for sufficiently large $t$. Property (a) is thus satisfied, and since $\left(b^{\prime \prime}\right)$ holds by definition, $\Lambda$ is an attracting set.

3.3. Irreducibility. A definition of irreducible attracting sets will be given in Sect. 4.5. 


\section{Attractors}

In this section we assume that $M$ has a uniform structure given by a metric ${ }^{5}$ dist. We assume also that $(x, t) \mapsto f^{t} x$ is continuous on $M \times\{t\}$. A curve (not necessarily continuous), i.e. a family $\left(x_{t}\right)_{t \in\left[t_{0}, t_{1}\right]}$ (with $t_{1} \geqq t_{0}$ ) of points of $M$ is called an $\varepsilon$ pseudoorbit if

$$
\operatorname{dist}\left(f^{\beta} x_{t+\alpha}, f^{\alpha+\beta} x_{t}\right)<\varepsilon
$$

whenever $\alpha, \beta \geqq 0, \alpha+\beta \leqq 1$ and $t, t+\alpha \in\left[t_{0}, t_{1}\right]$. In the discrete time case this simply means dist $\left(f x_{t}, x_{t+1}\right)<\varepsilon$ for $t=t_{0}, \ldots, t_{1}-1$ (see Bowen [3] and references quoted there). We say that the above $\varepsilon$ pseudoorbit is of length $t_{1}-t_{0}$ and goes from $x_{t_{0}}$ to $x_{t}$. Putting together two $\varepsilon$ pseudoorbits, the one going from $a$ to $b$ and of length $T$, the second going from $b$ to $c$ and of length $T^{\prime}$, we obtain a $2 \varepsilon$ pseudoorbit (an $\varepsilon$ pseudoorbit in the discrete time case) of length $T+T^{\prime}$ and going from $a$ to $c$. We introduce now a relation $a \succ b$, which we may read " $a$ goes to $b$ " and which means roughly that there is a slightly perturbed orbit going from $a$ to $b$. In fact, for $a, b \in M$ we write $a>b$ if, for arbitrarily small $\varepsilon>0$, there is an $\varepsilon$ pseudoorbit going from $a$ to $b$. We note the following easy fact.

4.1. Proposition. The relation $\succ$ is a preorder which means that it is reflexive $(a \succ a)$ and transitive $(a \succ b$ and $b \succ c$ imply $a \succ c)$.

The relation $\succ$ is closed (i.e. if $x$ and $y$ tend to $a$ and $b$ respectively, and if $x>y$ then $a \succ b$ ).

Write $a \sim b$ if $a \succ b$ and $b \succ a$. Since $\succ$ is a preorder, $\sim$ is an equivalence relation and $\succ$ induces an order on the equivalence classes, which we denote by $[a] \geqq[b]$ if $a>b$. A minimal equivalence class will be called an attractor. We shall say that an equivalence class $[a]$ is a basic class if either $a$ is a fixed point or $\operatorname{card}[a]>1$.

The main source of the above definitions is Conley [9] (see also Hurley [17] ${ }^{6}$ ) but we have here a somewhat different terminology and emphasis. The union of the basic classes is Conley's chain recurrent set, which has a nice characterization in terms of attracting sets (Conley [9, p. 37]). A set $S$ is chain transitive if it is contained in a basic class, and a basic class is called a chain component. What we call an attracting set is an attractor in the sense of Conley; we prefer to reserve the name of attractors to sets satisfying an irreducibility condition ${ }^{7}$. Our attractors are the chain transitive quasiattractors of Hurley. Conley and Hurley restrict their attention to compact $M$ (and continuous time); we discuss here a more general situation in view of physical applications (for instance to the Navier-Stokes equation, where $M$ is a Banach space). Altogether, a number of results in this section are not very new (and also not very hard). These results will serve as a basis

5 Two metrics $d, d^{\prime}$ define the same uniform structure if, for each $\varepsilon>0$, there is $\delta>0$ such that $d(x, y)<\delta$ implies $d^{\prime}(x, y)<\varepsilon$ and $d^{\prime}(x, y)<\delta$ implies $d(x, y)<\varepsilon$. One could actually proceed with a Hausdorff uniform structure which is not necessarily associated with a metric. If $M$ is compact it has a unique uniform structure compatible with the topology. If $M$ is compact with a countable base for the topology, it has a metric compatible with the topology

6 Hurley obtains interesting results on the generic behavior of attracting sets

7 Our terminology for attractors and basic classes agrees with the accepted terminology in the Axiom $A$ case (Smale [35]) 
for the discussion of small random perturbations in Sect. 5 (which constitutes the more original part of the present paper).

4.2. Proposition. Every equivalence class $[a]$ is closed.

$[a]$ is a basic class if and only if, for each $\varepsilon>0$, there is an $\varepsilon$ pseudoorbit of length $>1$ going from a to a.

The union of all basic classes is closed. An attractor is a basic class.

If $a$ is a nonwandering point, $[a]$ is a basic class.

If $[a]$ is a basic class, $f^{t}[a] \subset[a]$ for all $t$.

These are again easy results. For instance, if $[a]$ is a basic class there exists a long $\delta$ pseudoorbit with small $\delta$ going from $a$ to $a$, and therefore there are $\varepsilon$ pseudoorbits from $a$ to $f^{t} a$ and from $f^{t} a$ to $a$. Thus $f^{t} a \in[a]$, hence $f^{t}[a] \subset[a]$.

4.3. Corollary. If an $\left(f^{t}\right)$ ergodic probability measure @ has compact support ${ }^{8}$ this support is contained in a basic class.

The algebra of real continuous functions on supp $\varrho$ is separable. Therefore, by the ergodic theorem, there is $a \in \operatorname{supp} \varrho$ such that supp $\varrho$ is the closure of $\left\{f^{t} a\right\}$. Since $f^{t} a \in[a]$ by Proposition 4.2, supp $\varrho \subset[a]$.

4.4. Theorem. Let $\Lambda$ be a compact attracting set. We assume that $\Lambda$ has a neighborhood on which $f^{1}$ is uniformly continuous (this condition is satisfied when $M$ is locally compact, or when $M$ is a Banach manifold and $f^{1}$ is differentiable of class $\left.C^{1}\right)$.

(a) If $a \in \Lambda$ and $a \succ b$, then $b \in \Lambda$.

(b) If $a$ is in the basin of attraction of $\Lambda$ and $[a]$ is a basic class, then $[a] \subset \Lambda$ and $f^{t}[a]=[a]$ for all $t^{9}$.

(c) If $a$ is in the basin of attraction of $\Lambda$, there is at least one attractor [b] such that $a \succ b$, and we have necessarily $[b] \subset \Lambda$.

(d) A class [a]C $A$ is an attractor if and only if, for every neighborhood $V$ of $[a]$, there is a neighborhood $V^{\prime}$ of $[a]$ such that $x \in V^{\prime}$ and $x>y$ imply $y \in V$.

(e) Given a compact set $K \subset \Lambda$, we know that $K^{*}=\bigcup_{z \in K}[z]$ is compact (by Proposition 4.1). For all $\theta>0$ one may choose $\varepsilon>0$ such that if $y>x \in K$ and there is an $\varepsilon$ pseudoorbit from $x$ to $y$, then $\operatorname{dist}\left(y, K^{*}\right)<\theta$.

(f) $\Lambda$ is an attractor if and only if it is a basic class.

Let $\Omega$ be the neighborhood of $\Lambda$ on which $f^{1}$ is assumed to be uniformly continuous. There exist $\delta>0$ and a neighborhood $\Omega^{\prime}$ of $\Lambda$ such that the $\delta$-neighborhood ${ }^{10}$ of $\Omega^{\prime}$ is contained in $\Omega$. For any fundamental neighborhood $\tilde{U}$ of $\Lambda$ we know that $f^{t} \tilde{U}$ will be in $\Omega^{\prime}$ for sufficiently large $t$. We may thus choose a smaller fundamental neighborhood $U$ such that $f^{n} U \subset \Omega^{\prime}$ for all integers $n \geqq 0$.

Take a neighborhood $V$ of $\Lambda$, contained in $U$. There is $\delta^{\prime}>0$ and a neighborhood $V^{\prime}$ of $\Lambda$ such that the $\delta^{\prime}$-neighborhood of $V^{\prime}$ is contained in $V$. Using

8 If we had a uniform structure, but not necessarily a metric, we should assume here that supp $\varrho$ is a metrizable compact set

9 If $[a]$ is a basic class and $\left\{f^{t}\right\}$ is a group, then $f^{t}[a]=[a]$ already as a consequence of $f^{t}[a] \subset[a]$ (Proposition 4.2)

10 The $\delta$-neighborhood of $\Omega^{\prime}$ is the union of all balls of radius $\delta$ centered at a point of $\Omega^{\prime}$ 
the compactness of $\Lambda$ and $[0,1]$ and the continuity of $(x, t) \mapsto f^{t} x$, we can also choose a neighborhood $V^{\prime \prime}$ of $\Lambda$ such that $f^{t} V^{\prime \prime} \subset V^{\prime}$ when $0 \leqq t \leqq 1$. A $\delta^{\prime}$ pseudoorbit of length $t \leqq 1$ starting in $V^{\prime \prime}$ ends therefore in $V$. We take finally $\delta^{\prime \prime}>0$ and a neighborhood $V^{\prime \prime \prime}$ of $\Lambda$ such that the $\delta^{\prime \prime}$-neighborhood of $V^{\prime \prime \prime}$ is contained in $V^{\prime \prime}$.

Since $U$ is a fundamental neighborhood of $\Lambda$ there is a positive integer $N$ such that $f^{t} U \subset V^{\prime \prime \prime}$ for $t \geqq N$. In particular

$$
f^{n} U \subset V^{\prime \prime \prime} \text { for } n=N, N+1, \ldots, 2 N-1 \text {. }
$$

Using now the uniform continuity of $f^{1}$ we may choose $\varepsilon>0$ such that all $\varepsilon$ pseudoorbits of length $N, N+1, \ldots$, or $2 N-1$ starting at a point of $U$ end at a point of $V^{\prime \prime}$. It is no restriction to assume also $\varepsilon<\delta^{\prime}$, so that all $\varepsilon$ pseudoorbits of length $t \in[N, 2 N]$ starting at a point of $U$ end at a point of $V$. Since we assumed $V \subset U$, we find that all $\varepsilon$ pseudoorbits of length $t \geqq N$ starting at a point of $U$ end at a point of $V$.

(a) If $a \in \Lambda$, we may write $a=f^{N} c$, where $c \in \Lambda$. Thus, for every $\varepsilon$ pseudoorbit of length $t \geqq 0$ from $a$ to $b$ there is an $\varepsilon$ pseudoorbit of length $N+t \geqq N$ from $c$ to $b$, hence $b \in V$. We have thus shown that $a \succ b$, with $a \in \Lambda$ implies $b \in \Lambda$.

(b) If $a$ is in the basin of attraction of $\Lambda$ and [a] is a basic class we may construct a long $\varepsilon$ pseudoorbit with small $\varepsilon$ going from $a$ to $a$. We may assume that the pseudoorbit enters $U$, and therefore that $a$ is in any neighborhood $V$ of $A$, thus $a \in 1$. If $x$ is the point on the pseudoorbit at length $t$ before the end we may (by compactness) assume that $x$ tends to a limit $c \in \Lambda$ when the length of the pseudoorbit tends to $\infty$ and $\varepsilon$ tends to 0 . Then $f^{t} c=a$ and $c \sim a$, so that $f^{t}[a] \supset[a]$. Since $f^{t}[a] \subset[a]$ by Proposition 4.2, we have $f^{t}[a]=[a]$ for all $t$.

(c) We now want to prove that, for $a$ in the basin of attraction of $\Lambda$ there is an attractor $[b]$ such that $a \succ b$, and $b$ is necessarily in $\Lambda$. The problem reduces to the case where $a \in U$ and, according to earlier discussion, we only have to show that the set $\{[x]: a \succ x\}$ has a minimal element. Consider a totally ordered set $X$ of classes $\left[x_{\alpha}\right]$ with $a \succ x_{\alpha}$. If we can show that $X$ is bounded below, the existence of a minimal element of $\{[x]: a>x\}$ will follow from Zorn's lemma. The case where $x_{\alpha}=f^{t_{\alpha}} x$ for all $\alpha$ and the $t_{\alpha} \geqq 0$ are bounded is trivial. Otherwise, by our earlier discussion, the distance $d\left(x_{\alpha}, \Lambda\right)$ tends to 0 and we may choose $y_{\alpha} \in \Lambda$ with $d\left(x_{\alpha}, y_{\alpha}\right)$ tending to zero. If $b$ is the limit of a suitable subnet of $\left(y_{\alpha}\right)$, the corresponding subnet of $\left(x_{\alpha}\right)$ tends to $b$ and it follows that $x_{\alpha}>b$ for all $a$, hence $[b]$ is a lower bound for $X$.

(d) Let $V$ be a neighborhood of $[a] \subset \Lambda$ and suppose that there is no neighborhood $V^{\prime}$ of $[a]$ such that $x \in V^{\prime}$ and $x>y$ imply $y \in V$. Then one can find $\left(x_{\alpha}\right),\left(y_{\alpha}\right)$ such that $x_{\alpha} \succ y_{\alpha}, d\left(x_{\alpha},[a]\right) \rightarrow 0$, and $y_{\alpha} \notin V$. By going to a subnet one may assume that $x_{\alpha} \rightarrow x \in[a], y_{\alpha} \rightarrow y \in \Lambda \backslash V$. Thus $x>y \notin V$ and [a] cannot be an attractor. Conversely, if $[a]$ is not an attractor one can find $x \in[a]$ and $y \notin[a]$ with $x>y$. Therefore if $V_{\alpha} \ni y$ there is no $V^{\prime}$ with the listed properties.

(e) Otherwise one could find sequences $\left(x_{n}\right),\left(y_{n}\right)$ with $x_{n} \in K$ such that $y_{n} \succ x_{n}$, there is a $\frac{1}{n}$ pseudoorbit from $x_{n}$ to $y_{n}$, and the distance from $y_{n}$ to $K^{*}$ is $\geqq \theta$. One may assume that $x_{n} \rightarrow \bar{x} \in K, y_{n} \rightarrow \bar{y} \in \Lambda$, hence $\bar{x} \sim \bar{y}$ so that $\bar{y} \in K^{*}$ in contradiction with $\operatorname{dist}\left(\bar{y}, K^{*}\right) \geqq \theta$. 
(f) If $\Lambda$ is an attractor, it is a basic class by Proposition 4.2. On the other hand if $\Lambda$ is a basic class, it is an attractor in view of (c).

4.5. Definition of Irreducible Attracting Sets. In the situation of Theorem 4.4, it is natural to say that an attracting set is irreducible if it is a basic class (or equivalently an attractor). In particular, an attracting set is irreducible if it is positively transitive ${ }^{11}$ or if it is the support of an ergodic probability measure (see Corollary 4.3).

4.6. Remark. In the situation of Theorem 4.4, if $[a]$ is an intersection of attracting sets, then $[a]$ is an attractor [use Theorem 4.4(a)]. Conversely, every attractor is the intersection of the attracting sets containing it, when $\left\{f^{t}\right\}$ is a group, or when $A$ has a neighborhood $U$ such that $f^{t} U$ is relatively compact for large $t$ (in that case we may choose $U, S$ compact $\subset U$, and s such that $f^{t} U \subset S$ for $t \geqq s$ ). This result is basically due to Conley [9, p. 37], and motivates Hurley's definition of chain transitive quasiattractors. To prove the result, let $[a]$ be an attractor, and $U_{\varepsilon}$ the interior of the set of points $x$ such that there is an arbitrary long $\varepsilon$ pseudoorbit from $a$ to $x$. Therefore $U_{\varepsilon}$ contains the $\varepsilon$ neighborhood of $f^{t} U_{\varepsilon}$ for $t \geqq 1$. We write $K_{\varepsilon}=\bigcap_{t \geqq 0} f^{t} U_{\varepsilon}$. We assume $\varepsilon$ small so that $U_{\varepsilon}$ is contained in a fundamental neighborhood of $\Lambda$, and therefore $K_{\varepsilon} \subset \Lambda$; by our assumptions we may also assume that clos $f^{t} U \subset S$ closed $\subset U$ for $t \geqq S$, and $f^{t} S$ is closed for $t \geqq 0$. In particular

$$
K_{\varepsilon}=\bigcap_{t \geqq 0} f^{t} S
$$

and $K_{\varepsilon}$ is compact. For open $V \supset K_{\varepsilon}$ we claim that $f^{t} U_{\varepsilon} \subset V$ for $t$ large enough. Otherwise, let $x_{n} \in f^{t_{n}} S \backslash V$ with $t_{n+1}-t_{n} \geqq S$ so that $f^{t_{n+1}} S \subset f^{t_{n}} S$. Since $U_{\varepsilon}$ is contained in a fundamental neighborhood of $\Lambda$, we may take $x_{n} \rightarrow x \in \Lambda \backslash V$ and we have $x \in \cap f^{t} S=K_{\varepsilon}$ in contradiction with $x \notin V . K_{\varepsilon}$ is thus an attracting set, and

proving our assumption.

$$
[a] \subset \bigcap_{\varepsilon>0} K_{\varepsilon} \subset \bigcap_{\varepsilon>0} U_{\varepsilon}=[a]
$$

\section{Small Random Perturbations}

The idea of this section is to show that for a dynamical system submitted to small random perturbations, the motion is asymptotically concentrated on attractors. We shall be able to prove this (with some qualifications) for discrete time dynamical systems with bounded perturbations satisfying an "absolute continuity" condition.

5.1. Diffusions with Compact Support. Let $M$ be a metric space ${ }^{12}$ and $f: M \mapsto M$ be a continuous map, generating a discrete time dynamical system $\left(f^{t}\right)$. Let $\varepsilon>\delta>0$. We say that an affine map $F$ from the space of probability measures with compact support in $M$ to itself is an $(\varepsilon, \delta)$-diffusion associated with $f$ if the following conditions are satisfied ${ }^{13}$.

11 The definition has been given in Sect. 2 (D). For a brief discussion see Ruelle [29, Appendix A.2]

12 For the essential results of this section it would be sufficient to assume that we have a metrizable uniform structure

$13 \delta$ denotes the unit mass at $x, B_{x}(\varepsilon)$ the open ball of radius $\varepsilon$ centered at $x$, and $\bar{B}_{x}(\varepsilon)$ its closure 
(a) $\operatorname{supp} F \delta_{x} \subset f \bar{B}_{x}(\varepsilon)$.

(b) $\operatorname{supp} F \delta_{x} \supset f \bar{B}_{x}(\delta)$.

(c) If $\phi: M \mapsto \mathbb{R}$ is continuous, then $x \mapsto\left(F \delta_{x}\right) \phi$ is continuous, and $(F \mu) \phi$ $=\int \mu(d x)\left[\left(F \delta_{x}\right) \phi\right]$. In particular

$$
\operatorname{supp} F \mu=\text { closure } \bigcup_{x \in \operatorname{supp} \mu} \operatorname{supp} F \delta_{x} .
$$

(d) If $\phi: M \mapsto \mathbb{R}$ is continuous, the set $\left\{\left(F \delta_{y}\right) \phi: y \in \bar{B}_{x}(\delta)\right\}$ is a closed interval.

Property (d) holds if one can find a weaker topology on $\bar{B}_{x}(\delta)$ such that this set becomes compact, and the map $y \mapsto\left(F \delta_{y}\right)$ is continuous with respect to the compact topology on $\bar{B}_{x}(\delta)$ and the vague topology on measures.

5.2. Remark. The above formulation corresponds to thinking of $F$ as $f$ preceded by a small diffusion. This permits the treatment of measures with compact support in the infinite dimensional case. In the finite dimensional (locally compact) case one could take $F$ to be $f$ followed by a small diffusion.

Condition (a) expresses that the perturbations are bounded, while (b) is an "absolute continuity" type condition, without which one cannot expect the next result to be true.

5.3. Theorem. Let $\left(f^{t}\right)$ be a discrete time dynamical system, and assume that the class $[a]$ is not an attractor. For sufficiently small $\varepsilon$, if $F$ is an $(\varepsilon, \delta)$-diffusion associated with $f$, and $v$ a probability measure with compact support in $M$, then

$$
\lim _{t \rightarrow \infty}\left(F^{t} v\right)\left(B_{a}(\delta)\right)=0 .
$$

In particular, if $v_{\infty}$ is a vague limit ${ }^{14}$ of the measures $F^{t} v$, then a£supp $v_{\infty}$.

Let $a \succ b$ and $b \notin[a]$. Since $b \notin[a]$, for $\varepsilon$ small enough there are no $2 \varepsilon$ pseudoorbits from $b$ to $a$. Therefore there is no pseudoorbit from $B_{f b}(\varepsilon)$ to $B_{a}(\varepsilon)$. In particular there is no $\varepsilon$ pseudoorbit from $B_{f b}(\varepsilon)$ to $B_{a}(\delta)$.

If $x \in \bar{B}_{a}(\delta)$, (b) gives supp $F \delta_{x} \supset f \bar{B}_{x}(\delta) \ni f a$. Since $a \succ b$ there is a $\delta$ pseudoorbit going from $a$ to $b$, say $\left(x_{0}, x_{1}, \ldots, x_{m}\right)$ with $x_{0}=a, x_{m}=b$. We have just seen that $f x_{0} \in \operatorname{supp} F \delta_{x}$. Using (c) we obtain also

$$
f x_{1} \in \operatorname{supp} F^{2} \delta_{x}, \ldots, f x_{m} \in \operatorname{supp} F^{m+1} \delta_{x} .
$$

If $\phi$ is a continuous function with values in $[0,1]$ and support in $\bar{B}_{f b}(\varepsilon)$, and if $\phi(f b)>0$, we have thus

$$
\left(F^{m+1} \delta_{x}\right)(\phi)>0 \text { for all } x \in \bar{B}_{a}(\delta) .
$$

In view of $(d)$, there is $\beta>0$ such that

$$
\left(F^{m+1} \delta_{x}\right)(\phi) \geqq \beta \quad \text { for } \quad x \in \bar{B}_{a}(\delta)
$$

and therefore, if $\mu$ is a positive measure, (c) gives

$$
\left(F^{m+1} \mu\right)\left(B_{f b}(\varepsilon)\right) \geqq\left(F^{m+1} \mu\right)(\phi) \geqq \beta \mu\left(B_{a}(\delta)\right) .
$$

Let $y \in B_{f b}(\varepsilon)$. In view of (a) and (c), each point of $\operatorname{supp} F^{n} \delta_{y}$ is of the form $f y_{m}$ for some $2 \varepsilon$ pseudoorbit $\left(b, y, y_{1}, \ldots, y_{n}\right)$. Since there is no $2 \varepsilon$ pseudoorbit from $b$ to $a$, $\operatorname{supp} F^{n} \delta_{y}$ must be disjoint from $B_{a}(\delta)$.

$14 v_{\infty}$ is a vague limit of $F^{t} v$ if, for all $\alpha>0, N$ and continuous $\phi_{1}, \ldots, \phi_{N}: M \mapsto \mathbb{R}$, there are arbitrary large $t$ with $\left|\left(F^{t} v\right)\left(\phi_{i}\right)-v_{\infty}\left(\phi_{i}\right)\right|<\alpha$ for $i=1, \ldots, N$ 
Given the probability measure $v$, suppose now that

$$
F^{t} v\left(B_{a}(\delta)\right) \geqq \gamma>0, \quad \text { for } \quad t=t_{1}, \ldots, t_{k} .
$$

Then, if $T \geqq t_{1}, \ldots, t_{k}$, we find

$$
\begin{aligned}
1 & \geqq\left(F^{T+m+1} v\right)\left(M \backslash B_{a}(\delta)\right) \\
& \geqq k \beta \gamma .
\end{aligned}
$$

From this (2) results immediately.

5.4. Diffusions with "large" Support. We have assumed above that $F \delta_{x}$ has small compact support. We discuss here briefly the case where $\operatorname{supp} F \delta_{x}$ is not small, for instance not compact ${ }^{15}$. We say that an affine map from probability measures to probability measures is an $(\varepsilon, \delta, \alpha)$ diffusion associated with $f$ if it satisfies

$$
\left(F \delta_{x}\right)\left(f \bar{B}_{x}(\varepsilon)\right)>1-\alpha
$$

and the conditions (b)-(d) of 6.1. For sufficiently small $\varepsilon$ an argument similar to the proof of Theorem 5.3, with the same choice of $\beta$, yields

$$
\limsup _{N \rightarrow \infty} \frac{1}{N} \sum_{t=0}^{N-1}\left(F^{t} v\right)\left(B_{a}(\delta)\right) \leqq \frac{\alpha}{\beta} .
$$

In some cases one may assume that $\alpha / \beta \rightarrow 0$, and obtain invariant measures which do not contain $a$ in their support ${ }^{16}$, but a simple and general result seems difficult to obtain. Diffusions with "large" support are important because they occur naturally in the continuous time case.

5.5. Discussion. We return to the discussion of an $(\varepsilon, \delta)$ diffusion associated with a map $f$, and assume that the compact support of the probability measure $v$ is contained in the domain of attraction of a compact attracting set $\Lambda$ such that $\Lambda$ has a neighborhood on which $f$ is uniformly continuous ${ }^{17}$. Theorems 4.4 and 5.3 are then both applicable.

Let $A$ be the union of all attractors contained in $\Lambda, \bar{A}$ its closure, and $A^{*}=\bigcup_{z \in A}[z]$. If $a$ is in the basin of attraction of $\Lambda$ we may, by Theorem 4.4(c) assume that $a \succ b \in A$. Given $\theta>0$ we may also, by Theorem 4.4(e), choose $\varepsilon$ such that, if $a$ is not in the $\theta$-neighborhood of $A^{*}$, and if $a>b \in A$, there is no $2 \varepsilon$ pseudoorbit from $b$ to $a$. With $a, b, \varepsilon$ so chosen in the proof of Theorem 5.3, (2) holds. In other words, given $\theta>0$, for sufficiently small $\varepsilon$, if $F$ is an $(\varepsilon, \delta)$ diffusion associated with $f$, and $v$ a probability measure with compact support in the basin of attraction of $\Lambda$,

$$
\lim _{t \rightarrow \infty}\left(F^{t} v\right)\left(B_{a}(\delta)\right)=0
$$

15 We may work with the class of probability measures $v$ such that $v$ is carried by a countable union of compact subsets of $M$. This gives a natural definition of support, and the possibility to integrate bounded continuous functions

16 Let $F=\theta F_{1}+(1-\theta) F_{2}$ where $F_{1}$ is an $(\varepsilon, \delta)$ diffusion, then $\alpha / \beta \rightarrow 0$ when $\theta \rightarrow 1$

17 One might assume instead that $v$ is carried by a countable union of compact subsets of the basin of attraction of $\Lambda$ 
for some $\delta=\delta(a)$ and all $a$ not in the $\theta$ neighborhood of $A^{*}$. Vague limits of $F^{t} v$ when $t \rightarrow \infty$ and then $\varepsilon \rightarrow 0$ can be defined, and are thus probability measures on $\Lambda$ with support in $A^{*}$. We have established the following Corollary of Theorem 5.3 (it really is a corollary of the proof of this theorem).

5.6. Corollary. Let $\left(f^{t}\right)$ be a discrete time dynamical system, $F$ an $(\varepsilon, \delta)$ diffusion associated with $f$, and $v$ a probability measure with compact support in the basin of attraction of an attracting set $\Lambda$. We assume that $\Lambda$ has a neighborhood on which $f$ is uniformly continuous. We denote by $A$ the union of all attractors contained in $\Lambda$, by $\bar{A}$ the closure of $A$, and we write $A^{*}=\bigcup_{z \in A}[z]$.

For every neighborhood $\Theta$ of $A^{*}$, if $\varepsilon$ is small enough

$$
\lim _{t \rightarrow \infty} F^{t} v(M \backslash \Theta)=0
$$

In particular any vague limit $\lim _{\varepsilon \rightarrow 0} \lim _{t \rightarrow \infty} F^{t} v$ has support in $A^{*}$.

It may be that one can replace $A^{*}$ by a smaller set in this corollary. In any case, if $A$ is closed (for instance if the number of attractors is finite) then $A^{*}=A$ and one obtains measures with support asymptotically in $A$.

\section{Further Remarks}

6.1. Examples. In the study of maps of the interval, Feigenbaum ${ }^{18}$ has encountered a remarkable phenomenon of accumulation to a limit of an infinite sequence of bifurcations. For $n=0,1, \ldots$ an attracting periodic orbit of period $2^{n}$ becomes repulsive, while an attracting periodic orbit of period $2^{n+1}$ is created. As $n \rightarrow \infty$, a Cantor set is produced, which is an attractor in the sense of Sect. 4. This Feigenbaum Cantor set is not an attracting set because there are repulsive periodic orbits of period $2^{n}$ arbitrarily close to it.

Starting with the usual construction of a Cantor set in $[0,1]$ by successive removal of the "middle third" intervals, we obtain a dynamical system by introducing a repelling fixed point in each "removed" interval. With respect to this dynamical system, the Cantor set consists of fixed points, each of which is an attractor. In this case the set of attractors is thus uncountable (cf. Hurley [17, 3.4]).

In Sect. 2 we introduced the example of the vector field $X(x)=-x^{4} \sin \frac{\pi}{x}$. Modifying this to $X(x)=-e^{1 / x}$ for $x<0$ we see that the points $1 / n$ for $n$ odd are attractors; their limit 0 is however not an attractor. The set of attractors need thus not be closed.

On the circle $\mathbb{R}(\bmod 2 \pi)$ consider the vector field $X(x)=1-\cos x$. The whole circle is an attractor, but only 0 is a nonwandering point. An attractor may thus contain wandering points, and in particular be different from the support of any ergodic probability measure.

18 See Feigenbaum [10-12], Misiurewicz [24], Collet et al. [8], Collet and Eckmann [7], Campanino and Epstein [6], and Lanford [2] 
6.2. Stable and Unstable Manifolds. Suppose for simplicity that $\left(f^{t}\right)$ is a group, and define the stable manifold and the unstable manifold of a point $a$ respectively by

$$
\begin{aligned}
& \Psi_{a}^{-}=\left\{x: \lim _{t \rightarrow \infty} d\left(f^{t} x, f^{t} a\right)=0\right\} \\
& \Psi_{a}^{+}=\left\{x: \lim _{t \rightarrow \infty} d\left(f^{-t} x, f^{-t} a\right)=0\right\} .
\end{aligned}
$$

Then, if $[a]$ is a basic class, $\Psi_{a}^{-} \succ a \succ \Psi_{a}^{+}$. (In particular, if $[a]$ and $[b]$ are basic classes such that $\Psi_{a}^{+} \cap \Psi_{b}^{-} \neq \emptyset$, then $a \succ b$. If $a \sim b$ then $\Psi_{a}^{+} \cap \Psi_{b}^{-} \subset[a]$. If $[a]$ is an attractor, then $\Psi_{a}^{+} \subset[a]$.) While this result is true quite generally, it is particularly interesting for differentiable dynamical systems (diffeomorphisms or flows), because $\Psi_{a}^{ \pm}$is then often an immersed differentiable manifold, of which one has some understanding ${ }^{19}$.

6.3. Axiom A Dynamical Systems. Let $\left(f^{t}\right)$ (with $t \in \mathbb{Z}$ or $t \in \mathbb{R}$ ) be a dynamical system satisfying the Axiom $A$ and No Cycle Conditions ${ }^{20}$. Then the Axiom $A$ basic sets and attractors are just what we have called basic classes and attractors. There is a finite number of basic sets, conventionally ordered by the same relation which we have introduced on basic classes. Axiom $A$ systems are the prototype which one tries to imitate to obtain a general theory of differentiable dynamical systems; this is true in particular for the study of asymptotic measures, which we discuss now.

6.4. Asymptotic Measures and Strange Attractors. Call asymptotic measures the measures which occur as "zero noise" limits of stationary measures under small stochastic perturbations of a dynamical system $\left(f^{t}\right)$. We shall here assume that $M$ is a compact manifold, and that the kernel $F \delta_{x}$ of the diffusion $F$ is absolutely continuous with respect to Lebesgue measure on $M$. The discussion of Sect. 5 gives information on the support of the asymptotic measures. For Axiom $A$ diffeomorphisms and flows (with No Cycle), one has information on the measures themselves ${ }^{21}$. There is just one asymptotic measure per attractor; it is characterized by having conditional probabilities on unstable manifolds which are absolutely continuous with respect to the Lebesgue measure on the unstable manifold. In the Axiom $A$ case one also knows the behavior of $f^{t} v$ for large $t$ near a nonattracting basic set: if $v$ has continuous density with respect to Lebesgue measure, the mass remaining in a neighborhood of the basic set decays exponentially ${ }^{22}$. If Axiom $A$ does not hold, one may still hope that asymptotic measures are absolutely continuous on unstable manifolds in many cases ${ }^{23}$, but exceptions are known. It has been shown by Pugh and Shub [26] that if there is a measure absolutely continuous on unstable manifolds, it is the ergodic average for initial points forming a set of Lebesgue measure $>0$ on the manifold.

It should be pointed out here that finding the asymptotic measures is an essentially deeper question than estimating their support. To see this, notice that our discussion in Sect. 5 uses only the topology of $M$ (assuming $M$ compact). On

19 See Smale [35], Pesin [25], and Ruelle [30, 31]

20 See Smale $[35,36]$ and Bowen [4]

21 See Sinai [34], Ruelle [27], Bowen and Ruelle [5], and Kifer [18]

22 The rate of decay is identified as a "pressure"; see Bowen and Ruelle [5] and Kifer [18]

23 This has been advocated in Ruelle [28] 
the other hand the asymptotic measures depend essentially on the differentiable structure. For instance the measure theoretic entropy of the asymptotic measure of an Anosov diffeomorphism varies when the diffeomorphism is perturbed ${ }^{24}$ in spite of structural stability.

Attractors present themselves to us mostly as experimental objects (from computer, physical or chemical experiments). These objects come equipped with an asymptotic measure and one may define a strange attractor by the fact that its asymptotic measure has a positive characteristic exponent. This means that $f^{t} x$ and $f^{t}(x+\Delta x)$ diverge exponentially (almost everywhere). It is not clear at this point if a definition of strange attractors can be given independently of the consideration of an asymptotic measure. This is an important question from a structural point of view as we have noted above.

6.5. Generation of Attractors by Digital Computers. When a long orbit $\left\{f^{t} x: t \in[0, N]\right\}$ is calculated by a digital computer, one has to take into account the existence of roundoff errors. The roundoff errors are bounded, and it is reasonable to assume that their distribution is in some sense absolutely continuous. The discussion of Sect. 5 therefore applies and it is very likely that computer generated pictures of "attractors" really represent attractors as defined here. (Notice that computers always study discrete time dynamical systems, even when solving differential equations.) One should however beware of the fact that very large values of $N$ may be necessary in some cases to reach the asymptotic behavior. Another fact to keep in mind is that, when acting on precisely the same numbers, the computer always makes precisely the same roundoff error. This violates the absolutely continuous independent distribution of errors which we have postulated, as soon as the computer comes back to a point already visited. Since a computer is a finite machine, such loops necessarily occur, but one might expect them to be extremely long. In reality, it has been observed by Levy [22] that relatively short loops (of length $\sim 10^{3}$ ) occur in moderate precision calculations of the Hénon attractor. To avoid this one should choose the precision such that the length of loops becomes large with respect to $N$.

6.6. Application to Physical Experiments. In the discussion of experiments with hydrodynamical systems (or chemical systems, etc.) one meets with several difficulties. The fact that the time parameter is continuous is not a serious problem in itself, but implies that the random fluctuations cannot be assumed to remain bounded (if they are uncorrelated at different times). Furthermore the distribution of fluctuations is poorly understood (there is a good theory of thermal fluctuations in equilibrium, but it does not easily extend outside equilibrium, see Fox [13]). Referring to Sect.5.4, one sees that the experimental data will be close to attractors provided the frequency of large fluctuations (measured by $\alpha$ ) is small in terms of the time needed to leave nonattractor basic sets (this is of the order of $\beta^{-1}$ ). This condition is difficult to verify in principle, even if it is clear that it will often be satisfied in practice.

24 For a hyperbolic toral automorphism the entropy of the asymptotic measure is equal to the topological entropy, but becomes strictly less by a small perturbation 


\section{References}

1. Ahlers, G.: Low-temperature studies of the Rayleigh-Bénard instability and turbulence. Phys. Rev. Lett. 33, 1185-1188 (1974)

2. Bourbaki, N.: Topologie générale (fascicule de résultats). Paris: Hermann 1953

3. Bowen, R.: Equilibrium states and the ergodic theory of Anosov diffeomorphisms. Lecture Notes in Mathematics, Vol. 470. Berlin, Heldelberg, New York: Springer 1975

4. Bowen, R.: On axiom $A$ diffeomorphisms. CBMS Regional Conference, Ser. 35. Providence RI: Am. Math. Soc. 1978

5. Bowen, R., Ruelle, D.: The ergodic theory of Axiom $A$ flows. Invent. math. 29, 181-202 (1975)

6. Campanino, M., Epstein, H.: On the existence of Feigenbaum's fixed point. Commun. Math. Phys. 79, 261-302 (1981)

7. Collet, P., Eckmann, J.-P.: Iterated maps on the interval as dynamical systems. Progress in physics, Vol. 1. Boston: Birkhäuser 1980

8. Collet, P., Eckmann, J.-P., Lanford, O.E.: Universal properties of maps of an interval. Commun. Math. Phys. 76, 211-254 (1980)

9. Conley, Ch.: Isolated invariant sets and the Morse index. CBMS Regional Conference, Ser. 38. Providence RI: Am. Math. Soc. 1978

10. Feigenbaum, M.J.: Quantitative universality for a class of nonlinear transformations. J. Stat. Phys. 19, 25-52 (1978)

11. Feigenbaum, M.J.: The universal metric properties of nonlinear transformations. J. Stat. Phys. 21, 669-706 (1979)

12. Feigenbaum, M.J.: The transition to a periodic behaviour in turbulent systems. Commun. Math. Phys. 77, 65-86 (1980)

13. Fox, R.F.: Hydrodynamic fluctuation theories. J. Math. Phys. 19, 1993-1999 (1978)

14. Gollub, J.P., Swinney, H.L.: Onset of turbulence in a rotating fluid. Phys. Rev. Lett. 35, 927-930 (1975)

15. Hénon, M.: A two-dimensional mapping with a strange attractor. Commun. Math. Phys. 50, 69-77 (1976)

16. Hopf, E.: A mathematical example displaying the features of turbulence. Commun. Pure Appl. Math. 1, 303-322 (1948)

17. Hurley, M.: Attractors: persistence and density of their basins. (to appear)

18. Kifer, Yu.I.: On small random perturbations of some smooth dynamical systems. Izv. Akad. Nauk SSSR Ser. Mat. 38, 1091-1115 (1974). English translation: Math. USSR Izv. 8, 1083-1107 (1974)

19. Kifer, Yu.I.: Stochastic stability of the topological pressure. J. Anal. Math. 38, 255-286 (1980)

20. Landau, L.D.: Turbulence. Dokl. Akad. Nauk SSSR 44, 339-342 (1944)

21. Lanford, O.E.: A computer-assisted proof of the Feigenbaum conjectures (to appear)

22. Levy, Y.: Some remarks about computer studies of dynamical systems (to appear)

23. Lorenz, E.N.: Deterministic nonperiodic flow. J. Atmos. Sci. 20, 130-141 (1963)

24. Misiurewicz, M.: Structure of mappings of an interval with zero entropy. Publ. Math. IHES 53, 5-16 (1981)

25. Pesin, Ya.B.: Invariant manifold families which correspond to non-vanishing characteristic exponents. Izv. Akad. Nauk SSSR Ser. Mat. 40, 1332-1379 (1976). English translation: Math. USSR Izv. 10, 1261-1305 (1975)

26. Pugh, C.C., Shub, M.: Differentiability and continuity of invariant manifolds (to appear)

27. Ruelle, D.: Am. J. Math. 98, 619-654 (1976)

28. Ruelle, D.: Dynamical systems with turbulent behavior. Mathematical problem in theoretical Physics. Lecture Notes in Physics, Vol. 80, pp. 341-360. Berlin, Heidelberg, New York: Springer 1978

29. Ruelle, D.: Thermodynamic formalism. Encyclopedia of Math. and its Appl. Vol. 5. Reading, Mass. : Addison-Wesley 1978

30. Ruelle, D.: Ergodic theory of differentiable dynamical systems. Publ. Math. IHES 50, 275-306 (1979)

31. Ruelle, D.: Characteristic exponents and invariant manifolds in Hilbert space. Ann. Math. (to appear)

32. Ruelle, D. : Differentiable dynamical systems and the problem of turbulence. Bull. Am. Math. Soc. (New Series) 5, 29-42 (1981) 